\title{
Body Fat Responses to a 1-Year Combined Exercise Training Program in Male Coronary Artery Disease Patients
}

\author{
Nuno M. Pimenta ${ }^{1,2}$, Helena Santa-Clara ${ }^{1}$, Luís B. Sardinhal and Bo Fernhall ${ }^{3}$
}

Objective: To analyze the body fat (BF) content and distribution modifications in coronary artery disease (CAD) patients in response to a 1-year combined aerobic and resistance exercise training (CET) program. Design and Methods: We followed two groups of CAD male patients for 12 months. One group consisted of 17 subjects ( $57 \pm 12$ years) who engaged in a CET program (CET group) and the other was a age-matched control group of 10 subjects ( $58 \pm 11$ years). BF content and distribution were measured through dual energy X-ray absorptiometry (DXA) at baseline and follow-up.

Results: We found no differences on body mass and BMI between baseline and end of follow-up in both groups but, in CET group, we found significant reductions in all analyzed BF depots, including total BF $(21.60 \pm 6.00$ vs. $20.32 \pm 5.89 \mathrm{~kg}, P<0.01)$, \% total BF (27.8 \pm 5.5 vs. $26.4 \pm 5.4 \%, P<0.05)$, trunk fat $(12.54 \pm 3.99$ vs. $11.77 \pm 4.01 \mathrm{~kg}, P<0.05)$, $\%$ trunk fat $(31.1 \pm 6.9$ and $29.2 \pm 7.1 \%, P<0.05)$, appendicular fat $(8.22 \pm 2.08$ vs. $7.72 \pm 2.037 \mathrm{~kg}, P<0.01), \%$ appendicular fat $(25.7 \pm 4.9$ and $24.5 \pm$ $4.9 \%, P<0.05)$, and abdominal fat $(2.95 \pm 1.06$ vs. $2.75 \pm 1.10 \mathrm{~kg}, P<0.05)$. Control group showed significant increase in appendicular fat $(7.63 \pm 1.92 \mathrm{vs} .8 .10 \pm 2.12 \mathrm{~kg}, P<0.05)$.

Conclusions: These results confirm the positive effect of CET on body composition of CAD patients, despite no changes in body mass or BMI. In this study, we observed no alterations on BF distribution meaning similar rate of fat loss in all analyzed BF depots. These results also alert for the limitations of BMI for tracking body composition changes.

Obesity (2013) 21, 723-730. doi:10.1038/oby.2012.150

\section{Introduction}

Obesity and higher body fat (BF) content are associated with higher risk of metabolic disorders, cardiac events, and related mortality and morbidity in healthy populations $(1,2)$. The accumulation of fat in specific fat depots, particularly in central or abdominal regions as well as the pattern of BF accumulation, seem to play a primary role in the link between $\mathrm{BF}$ and the related metabolic disorders and hazardous events in the general population $(3,4)$. Coronary artery disease $(\mathrm{CAD})$ patients are in considerably higher risk for recurrent cardiac events and death from cardiac causes (5). In CAD patients, the same association between BF content or distribution and respective increased risk of adverse health outcomes, as observed in general population, seem to be present $(6,7)$ even though there are conflicting results, especially concerning BF distribution (8).

Weight loss and $\mathrm{BF}$ reduction, as well as exercise training, are recommended for $\mathrm{CAD}$ patients and are recognized by many pre- eminent authors and institutions as an effective nonpharmacologic approach to reduce subjects risk in secondary prevention programs $(9,10)$. The effects of exercise training on body weight and composition is fairly well documented (11) but in CAD patients there is a limited amount of longitudinal intervention studies focusing on this matter. In spite of considerable methodological diversity, these studies are generally in agreement concerning the effectiveness of exercise in reducing total and most regional $\mathrm{BF}$, but there are some contradictory results in the literature concerning body weight and BMI $(8,12-19)$. To our knowledge only one study has investigated the effect of exercise training on BF distribution in CAD patients (8). The mentioned study used waist-to-hip ratio (WHR) and it allowed them to examine whether the distribution of BF throughout the body changed their pattern of accumulation in response to exercise and fat reduction in CAD patients.

There are some studies focusing on BF and weight modifications in $\mathrm{CAD}$ patients, using general measures of body composition, but few

\footnotetext{
${ }^{1}$ Exercise and Health Laboratory, Interdisciplinary Centre for the Study of Human Performance, Faculty of Human Kinetics, Technical University of Lisbon, Cruz-Quebrada, Portugal ${ }^{2}$ Sport Sciences School of Rio Maior, Polytechnic Institute of Santarém, Santarém, Portugal ${ }^{3}$ College of Applied Health Sciences, University of Illinois, Urbana-Champaign, Illinois, USA. Correspondence: Helena Santa-Clara (santaclara@fmh.utl.pt)
}

Disclosure: The authors declared no conflict of interest.

See the online ICMJE Conflict of Interest Forms for this article.

Received: 12 May 2011 Accepted: 14 May 2012 First published online by Nature Publishing Group on behalf of The Obesity Society 19 July 2012. doi:10.1038/oby.2012.150 
TABLE 1 Cardiovascular history and medications

\begin{tabular}{lcc}
\hline & CET $(n=17)$ & Control $(n=10)$ \\
\hline History & & \\
MI $(n(\%))$ & $13(76.5)$ & $6(60.0)$ \\
CABG $(n(\%))$ & $8(47.1)$ & $3(30.0)$ \\
PTCA $(n(\%))$ & $9(52.9)$ & $7(70.0)$ \\
Medication & & \\
$\beta$-Blockers $(n(\%))$ & $9(52.9)$ & $5(50.0)$ \\
Nitrates $(n(\%))$ & $7(41.2)$ & $4(40.0)$ \\
Calcium channel blockers $(n(\%))$ & $6(35.3)$ & $6(60.0)$ \\
Platelet inhibitors $(n(\%))$ & $15(88.2)$ & $8(80.0)$ \\
ACE inhibitors $(n(\%))$ & $4(23.5)$ & $2(20.0)$ \\
Diuretics $(n(\%))$ & $1(5.9)$ & $1(10.0)$ \\
\hline
\end{tabular}

$\mathrm{ACE}$, angiotensin-converting enzyme; CABG, coronary artery bypass grafting; $\mathrm{CET}$, combined exercise training; MI, myocardial infarction; PTCA, percutaneous transluminal coronary angioplasty.

focused on regional body composition. Studies investigating the changes occurred in BF distribution in response to exercise in this population are lacking. The primary purpose of the present study was to analyze the changes occurred in total and regional $\mathrm{BF}$ content and $\mathrm{BF}$ distribution of CAD patients in response to a 1-year combined aerobic and resistance exercise training program. We hypothesized that a combined aerobic and resistance exercise training (CET) program will result in the reduction of weight, overall $\mathrm{BF}$ and specific $\mathrm{BF}$ depots such as abdominal fat, resulting in changes in $\mathrm{BF}$ distribution.

\section{Methods and Procedures}

\section{Subjects}

This study was conducted at Technical University of Lisbon, Faculty of Human Kinetics. Two groups of CAD male patients were included in this study: one group of 17 subjects $(57 \pm 12$ years) who engaged in a CET program for 12 months (CET group) and another group of 10 age-matched subjects $(58 \pm 11$ years) who benefited only from standard care, including individually tailored cardiac medications and continued cardiologist counseling and supervision. Both groups included patients who had been diagnosed with at least one of the following: stable angina pectoris, myocardial infarction, percutaneous transluminal coronary angioplasty, and coronary artery bypass grafting. Patients' cardiovascular history and medications are expressed in Table 1. All subjects had been stratified as low risk for events during exercise and underwent phase II and/or III cardiac rehabilitation (9), including 6 months of aerobic training, after the cardiac event. Exclusion criteria included: unstable angina, uncontrolled arrhythmias, congestive heart failure, high resting systolic blood pressure $(>160 \mathrm{~mm} \mathrm{Hg}$ ), high resting diastolic blood pressure $(>100 \mathrm{~mm} \mathrm{Hg}$ ), exercise-induced angina, complex arrhythmias or more than $1 \mathrm{~mm}$ ST-segment depression, exerciseinduced hypotension, diabetes mellitus, and any condition that constituted an absolute restriction from exercise (9). This study was approved by the University Institutional Review Board and all participants signed an informed consent before being included in this study and undergoing any study procedure. All methods used in the present study complied with good ethics and Portuguese laws.

\section{Study design}

After prescreening 44 subjects fulfilled the inclusion criteria of this study. Four subjects withdrew from the present study alleging limited time availability for enrolment. Seventeen subjects were assigned to the CET group based on their residential proximity to the exercise facilities, considering this would potentially maximize compliance to the CET program. The subjects in the control group were selected at baseline based on their age, gender, and general body measures (weight and BMI) to constitute a comparable group. Logistic worries inhibited random assignment of the subjects into both groups. Subjects did not choose group assignment. Subjects from the control group were asked to continue their normal activities and at the time of the admission in the study they were not exercising regularly. All subjects were taking one or more of the following medication: $\beta$-blockers, nitrates, calcium channel blockers, platelet inhibitors, and angiotensin-converting enzyme inhibitors, with similar use among both groups. The baseline characteristics of the subjects from both groups are presented in Table 2.

\section{Body composition}

Body composition was assessed using Dual-Energy X-ray Absorptiometry (DXA) (Explorer W; Hologic, Waltham, MA; Fan beam mode) whole body scans and anthropometric measurements both at baseline and follow-up. Repeated measurements in 18 young adults showed a coefficient of variation (CV) of $1.7 \%$ for total BF mass and $1.5 \%$ for total $\% \mathrm{BF}$. All scans were made in the morning after an overnight 12-h fast. Quality control with spine phantom was made every morning, and with step phantom every week. By default DXA software (QDR for windows, version 12.4; Hologic, Waltham, MA) estimates the head, trunk, arms and legs, both left and right, regions, fat content, according to a three-compartment model (fat mass, lean tissue, and bone mass). The trunk region of interest $(\mathrm{ROI})(\mathrm{CV}=0.005 \%)$ includes chest, abdomen, and pelvis. Appendicular ROI $(\mathrm{CV}=0.004 \%)$ includes both arms plus both legs. All scan analyses were made by the same observer. All scans were submitted to additional analysis by ROI to assess fat content of the abdominal region ( Figure 1) $(\mathrm{CV}=0.01 \%)$. The upper and lower limits of the abdominal ROI were determined as the upper edge of the second lumbar vertebra and the lower edge of the fourth lumbar vertebra, respectively $(20,21)$. The sides' limits were determined as to include all trunk length, but exclude any upper limb scan area $(20,21)$. Absolute and relative BF content results were registered to the nearest $0.01 \mathrm{~kg}$ and $0.1 \%$, respectively.

Anthropometric measurements consisted of weight, height, and BMI. Body weight was measured to the nearest $0.1 \mathrm{~kg}$, and height was measured to the nearest $0.1 \mathrm{~cm}$, on a scale with an attached stadiometer (model 770; Seca, Hamburg, Deutschland), according to standard protocol (22). Both weight and height were used to calculate the subject's BMI, by dividing the weight, in kilogram, by the squared height, in meters $\left(B M I=\right.$ weight $(\mathrm{kg}) /$ height $\left.(\mathrm{m})^{2}\right)$. The rate of modification was calculated as the difference between 12-month and baseline results divided by baseline results times 100 $(((12$-month - baseline $) /$ baseline $) \times 100)$, for all variables absolute results and is presented in percentage to the nearest $0.01 \%$.

\section{Body fat distribution}

Body fat distribution variables were calculated using ratios between BF content absolute values of different fat depots, obtained by 
TABLE 2 Body composition and fat distribution at baseline and after 12 months intervention



Data are presented as mean \pm s.d.

$\mathrm{BF}$, body fat; CET, combined exercise training; FFM, fat-free mass; DBP, diastolic blood pressure; SBP, systolic blood pressure.

alndicates significant difference from baseline $(P<0.01)$. ${ }^{\text {In }}$ dicates significant difference from baseline $(P<0.05)$.

DXA, as done elsewhere (21). The trunk BF-appendicular BF ratio, also called trunk-extremity fat ratio (23) or central-peripheral fat mass ratio (24), was calculated as the trunk $\mathrm{BF}$ content divided by the sum of the BF content of the arms and legs, both left and right. The abdominal BF-trunk $\mathrm{BF}$ ratio was calculated as the fat content of the selected abdominal ROI divided by the trunk BF. The abdominal BF-total BF was calculated as the selected abdominal ROI fat content divided by the whole BF. Ratios were registered to the nearest 0.01 .

\section{Exercise testing}

All subjects underwent exercise assessment which included aerobic capacity and strength evaluation. Before the assessment of aerobic capacity subjects' blood pressure was measured after being seated quietly for $5 \mathrm{~min}$ in a chair, with feet on the floor, and arm supported at heart level. Blood pressure measurements were made by a trained operator using a standard sphygmomanometer (Maxi-Stabil 3 Mobile Stand; Welch Allyn, Skaneateles Falls, NY) and stethoscope (Master cardiology; 3M Littman, St Paul, MN). For the aerobic capacity assessment subjects performed a symptom-limited treadmill
(Q-65, Quinton; Cardiac Science, Bothell, WA) graded exercise test (GXT) using Bruce standard protocol (25). Gas analysis was performed during GXT using a MedGraphics CPX Ultima Cardio metabolic cart (Medical Graphics, St Paul, MN). Data on gas analysis was collected and recorded using Breeze Suite software (version 6.4.1; Medical Graphics). All GXT were monitored using a 12 lead electrocardiogram PC-based acquisition module (Welch-Allyn PCE210; Welch Allyn) and the data, including maximal heart rate, were monitored and recorded using Welch Allyn CardioPerfect software (Welch Allyn). Unless clinical test interruption criteria were observed, subjects exercised until at least two of the following test termination criteria were reached: (i) subjects volitional fatigue; (ii) respiratory exchange ratio reached 1.1 or higher; (iii) subjects reached maximal predicted heart rate; (iv) oxygen uptake did not increase in spite of increasing work load.

\section{Combined exercise training program}

The CET program consisted of three workouts/week of combined aerobic plus resistance exercise, on non-consecutive days, for 12 months. Workouts were held on a group class format. All sessions 

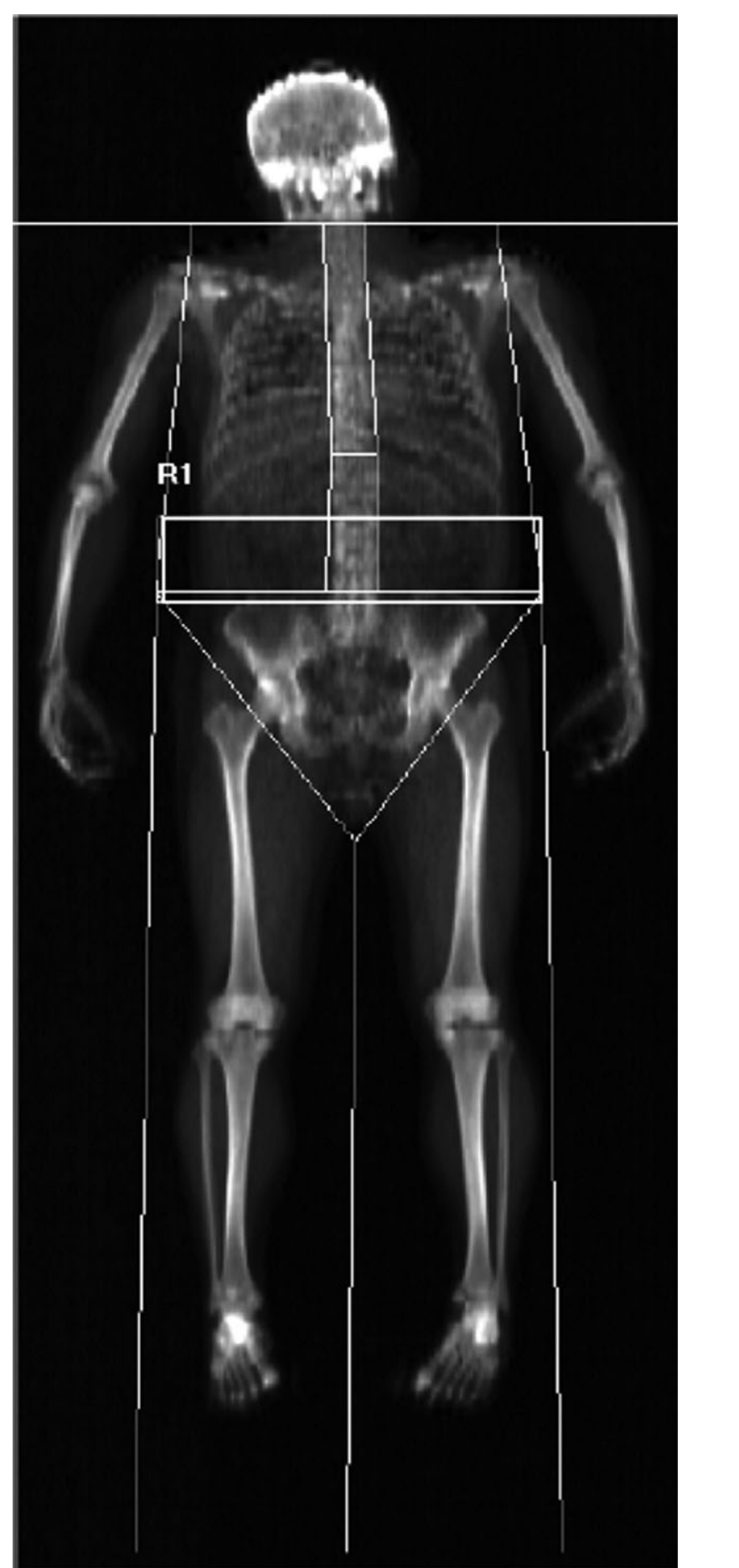

FIGURE 1 Image of a DXA scan showing the abdominal region of interest defined as the area within the upper edge of the second lumbar vertebra and the lower edge of the fourth lumbar vertebra. DXA, dual-energy X-ray absorptiometry.

included a 5-7 min warm-up, which included some calisthenics and stretch exercises, and a 5-7 $\mathrm{min}$ cool-down. The CET sessions included $30 \mathrm{~min}$ of aerobic exercise at an intensity of $60-70 \%$ of heart rate reserve (26) based on the maximal heart rate (HRmax) achieved on the GXT. Patients were provided with heart rate monitors (Pacer; Polar, Kempele, Finland) and were asked to adhere to prescribed intensity and they kept a daily record which included data on activity, duration, and heart rate during exercise. Aerobic exercise consisted of continuous upright dynamic exercises modes which included walking, jogging, cycle ergometry, rowing ergometry, stepping, and other such activities suitable for CAD patients. In the first 12 weeks, a progression was made in the exercise duration until all patients reached $30 \mathrm{~min}$ of aerobic exercise. The strength exercise training component $(20 \mathrm{~min})$ consisted of eight exercises (six upper body exercises: vertical traction, pectorals, chest press, deltoids, arm curl, triceps; and two lower body exercises: leg extension and leg press), two sets per exercise, 8-12 repetitions per set at $50 \%$ of 1 repetition maximum (RM), using variable resistance machines (Super Executive Line; Technogym, Gambettola, Italy). Patients' 1 RM was re-evaluated on all eight exercises on the 4th and 8th month of training, and adjustments were made on the mechanical load to keep the exercises relative intensity at $50 \%$ of 1 RM.

\section{Statistical methods}

Descriptive statistics are presented as mean \pm s.d. and range for all analyzed variables. The Gaussian distribution of the data was assessed with the Shapiro-Wilk goodness-of-fit test. The $t$-tests for related and independent samples were performed to compare results within and between groups, respectively. Corrected $t$-test $P$ values were used whenever variance homogeneity were not observed. Because BMI and trunk BF-appendicular BF ratio had a nonGaussian distribution in this setting, Mann-Whitney and Wilcoxon tests were applied for comparison between and within groups, respectively. The level of significance was set at $P<0.05$ (two-tailed). Statistical calculations were performed using the Predictive Analytics Software (PASW) Statistics version 18 (SPSS, Chicago, IL).

\section{Results}

Both groups had mean BMI that are considered as overweight, even though both groups included normal weight to obese CAD patients. There were no differences between groups in all baseline measures, including age as well as whole and regional body composition variables. Baseline results for both groups are presented in Table 2. The CET group patients completed $85 \%$ of the exercise sessions during the 12-month period, which was considered to be a high compliance to the CET program.

On a whole body analysis significant differences were found between baseline and end of 12-month intervention on total $\mathrm{BF}$ and $\%$ total $\mathrm{BF}$ in the CET group $(P=0.005$ and $P=0.013$, respectively), but not in control group. The general fat content reduction was observed in CET group despite no differences on general measures of body composition, such as weight and BMI, in both groups $(P=0.071$ and $P=0.48$ in CET group and $P=0.178$ and $P=$ 0.52 in control group, respectively for weight and BMI).

The regional body composition analysis showed significant changes in CET group either in central fat depots, such as on trunk fat and $\%$ trunk fat $(P=0.018$ and $P=0.018$, respectively), as in peripheral fat depots, such as appendicular fat and $\%$ appendicular fat 
TABLE 3 Body composition and fat distribution rate of modification

\begin{tabular}{|c|c|c|c|c|c|}
\hline \multirow[b]{2}{*}{ Variable } & \multicolumn{2}{|c|}{ CET $(n=17)$} & \multicolumn{2}{|c|}{ Control $(n=10)$} & \multirow[b]{2}{*}{$P$ value } \\
\hline & Mean \pm s.d. & Minimum-maximum & Mean \pm s.d. & Minimum-maximum & \\
\hline$\% \Delta$ Weight (\%) & $-0.15 \pm 1.95$ & -3.40 to 3.80 & $1.36 \pm 3.04$ & -3.70 to 6.20 & 0.099 \\
\hline$\% \Delta \mathrm{BF}(\%)$ & $-2.35 \pm 10.60$ & -27.74 to 17.97 & $7.21 \pm 14.02$ & -7.64 to 33.94 & $0.041^{\mathrm{a}}$ \\
\hline$\% \Delta \mathrm{FFM}(\%)$ & $1.18 \pm 3.13$ & -3.97 to 6.58 & $-1,67 \pm 3.56$ & -8.68 to 1.96 & $0.030^{\mathrm{a}}$ \\
\hline$\% \Delta$ Appendicular BF (\%) & $-4.15 \pm 7.82$ & -19.70 to 10.24 & $5.81 \pm 8.67$ & -4.30 to 21.04 & $0.003^{b}$ \\
\hline$\% \Delta$ Abdominal BF $(\%)$ & $-0.44 \pm 15.81$ & -33.07 to $35: 65$ & $14: 33 \pm 23.67$ & -13.08 to $58: 01$ & 0.060 \\
\hline$\% \Delta$ Trunk BF-appendicular BF ratio (\%) & $3.04 \pm 10.84$ & -21.40 to 27.46 & $3.17 \pm 11.10$ & -9.77 to 25.68 & $0: 714$ \\
\hline$\% \Delta$ Abdominal BF-trunk BF ratio (\%) & $-0.02 \pm 5.89$ & -9.04 to 11.17 & $3.82 \pm 5.46$ & -4.34 to 10.81 & 0.091 \\
\hline
\end{tabular}

Data are presented as mean \pm standard deviation and as minimum and maximum range limits.

BF, body fat; FFM, fat-free mass; CET, combined exercise training.

andicates significant difference from baseline $(P<0.05)$. ' Indicates significant difference from baseline $(P<0.01)$.

( $P=0.002$ and $P=0.049$, respectively), after the 12 -month intervention period. Control group showed no significant changes in central fat depots $(P=0.21$ and $P=0.301$ for trunk $\mathrm{BF}$ and $\%$ trunk BF, respectively) at the end of the follow-up period. Regarding peripheral fat depots, a significant increase after the 12 months period in the control group was found in absolute appendicular fat $(P=0.049)$ but not in relative appendicular fat $(P=0.059)$. When analyzing DXA-specific ROI, a significant decrease was found in CET group, between baseline and end of follow-up, in abdominal fat $(P=0.021)$ despite no significant differences in relative values $(P=0.061)$. Regarding the same ROI analysis, no differences were found between baseline and after 12 months in the abdominal region fat content variables in the control group $(P=0.21$ and $P=0.301$ for abdominal $\mathrm{BF}$ and $\%$ abdominal $\mathrm{BF}$ respectively). The whole and regional body composition results are presented in Table 2 .

Ratios between different whole and regional $\mathrm{BF}$ content variables were used to analyze subjects BF distribution. The trunk BF divided by appendicular BF showed that there is five times more BF accumulated in the trunk than in arms and legs together, in both groups both at baseline and at the end of follow up. The abdominal BF divided by the trunk $\mathrm{BF}$ showed that about $23 \%$ of all trunk fat is accumulated in the analyzed abdominal ROI, in both groups both at baseline and at the end of follow up. The abdominal BF divided by total BF showed that about $13-14 \%$ of all BF was accumulated in the same analyzed abdominal ROI, in both groups both at baseline and at the end of follow up. No significant differences were found in $\mathrm{BF}$ distribution variables between baseline and end of follow-up, within both groups. The results concerning BF distribution are presented in Table 2.

Significant differences were observed between groups in most of the analyzed $\mathrm{BF}$ content rate of modification variables such as $\triangle \mathrm{BF}(P=$ $0.041)$ and $\triangle$ appendicular $\mathrm{BF}(P=0.003)$. Although CET group showed a $1 \%$ reduction in mean trunk fat, while control group had a $10 \%$ increase, differences between groups were nonsignificant $(P=$ $0.099)$, as was the difference observed in $\Delta$ abdominal BF $(P=0.06)$. The absence of differences between groups found in the results for the rate of modification observed on weight and BMI $(P=0.99$ and $P=0.99$, respectively) confirms the previously mentioned absence of differences observed on these general measures of body composition within each group. The results relating to the rate of body composition modification variables are presented in Table 3 .

\section{Discussion}

The present study showed that a CETprogram was found beneficial for CAD patients' body composition but did not promote changes in $\mathrm{BF}$ distribution. Subjects from CET group reduced BF content in all analyzed fat depots, resulting in an overall significant $\mathrm{BF}$ reduction over the 12-month intervention period. On an opposite direction, control group, who benefited from standard care, showed a slight increase in appendicular fat content. Body composition profile improvement in response to cardiac rehabilitation and secondary prevention programs that included exercise have been observed in several other studies $(8,14-18,21,27)$.

Subjects from CET group did not change their weight or BMI but reduced their $\mathrm{BF}$. The effect of our CET program on the mentioned change in body composition was confirmed by the significant differences observed between both the intervention and the control group in the respective rate of modification. These results recall what have long been identified as BMI limitations in estimating body composition $(28,29)$. Other studies have reported similar observations in both long-term $(14,18)$ and short-term intervention studies $(8,12,15,17)$. Different results from the present study were found by Pierson and colleagues (17) in response to a 6-month intervention in the aerobic only exercise training group. Lavie and colleagues (15) also found different results from ours, concerning weight and total BF (using skinfolds), but only in the group of obese CAD patients, in response to a 14-week cardiac rehabilitation program that included aerobic only exercise training. Savage and colleagues (19) also observed significant reductions in weight, BMI, and both relative and absolute BF after a 4-month high caloric expenditure exercise training in a sample of obese CAD patients. More recently, a 
study that compared land with water exercise in a cardiac rehabilitation setting, also found body weight reductions along with the reduction in the sum of skinfolds, in response to a 4-month exercise intervention, regardless of the mode of exercise (30). These conflicting results may be partly explained by the subjects' baseline characteristics and/or by differences in the interventions' protocols. The studies that observed weight and BMI reductions studied samples of mainly obese subjects. Baseline BMI may be determinant for weight and BMI reductions to occur in cardiac rehabilitation setting exercise training, regardless of different intervention protocols and subjects ages. It has been shown that higher BMI subjects often lose both BF and lean body mass in response to weight loss programs that include exercise (31), whereas subjects with lower BMI may lose only BF and, in some cases, may even gain lean body mass (32). The absence of weight and BMI reduction, regardless of significant reductions in $\mathrm{BF}$, found in the present study in the CET group can be attributed to modest increases in subject's lean body mass resulting in body weight maintenance. Previous studies had already reported increases of lean body mass as a result of the combination of resistance training along with aerobic training, as compared to aerobic training alone or to controls $(17,18)$. Moreover, the studies that only included aerobic mode of exercise for CAD patients may need extended length interventions, as did Brubaker and colleagues' research (14), to produce significant body composition changes in a cardiac rehabilitation setting. Also higher intensity exercise seem to be more effective in reducing weight, BMI, and BF, regardless of caloric expenditure (33), but the cardiac rehabilitation studies we assessed used intensities based either on HRmax (up to $85 \%$ ) or on peak $\mathrm{VO}_{2}$ (up to $60 \%$ ) or on heart rate reserve (up to $80 \%$ ), as did our CET program intervention $(60-70 \%$ heart rate reserve). These disparities make it difficult to establish exercise protocol comparisons. Additional research should be conducted on this topic in CAD patients.

As was observed in the variables of general body composition, we also observed regional BF reductions in all analyzed BF depots in the CET group, contrasting with the control group that showed an increase in appendicular fat. A decrease in appendicular BF accompanied by a significant increase in central BF, would have been expected with aging, in accordance with previous findings in adults $(34,35)$. This outcome endorses the beneficial effect of our CET program intervention as compared with standard care. Even though we found significant differences in all BF depots, between baseline and end of follow up, within the CET group, contrasting with control group results, whole $\mathrm{BF}$ and regional fat depots content were not different between groups, both at baseline and at the end of followup (Table 2). To look deeper into the intervention effects, we compared the rate of modification of all analyzed variables between groups and we found significant differences in total and appendicular BF variables in CET group, as opposed to control group (Table 3). The differences between groups in the rate of trunk and abdominal fat content modification were not significant, even though, abdominal fat rate of modification showed close to marginal statistical difference between groups, and may as well suggest a trend towards higher fat reduction in CET group subjects as compared to controls. The present study contrasts with our previous observations in CAD patients that showed a significant decrease in trunk BF of CET group as compared to both aerobic only exercise training group and control group (18). Previous studies had already found $\mathrm{BF}$ reductions in regional fat depots and surrogates, including in waist circumference $(8,12,17,19)$, hip circumference $(16)$, and also in subcutaneous and visceral abdominal fat assessed by computed tomography (19). These discrepancies may be explained, once again, by the methodological diversity of the mentioned studies. The fact that there were no significant differences in the abdominal BF rate of modification between groups can be attributed to either the lack of time span of our study to identify the possible counter aging effect of our CET program or to the sample size that, even though showing a trend $(P=0.066)$ that may have clinical importance $(1 \%$ decrease while $10 \%$ augmentation in the abdominal BF of both CET and control groups respectively), were not able to identify significant statistical differences between groups. The effect of exercise training in the BF content of specific fat depots of CAD patients is surely not a closed issue and deserves further research.

To our knowledge no other study followed BF distribution response to exercise using ratios between different $\mathrm{BF}$ depots obtained by DXA, in CAD patients. Most of the studies we assessed focused only on general markers of body composition (weight, BMI, and total BF) and, not so commonly, on regional markers of body composition (waist circumference, trunk BF, appendicular BF). Almost absent in the literature is the analysis of $\mathrm{BF}$ distribution in $\mathrm{CAD}$ patients intervention studies. Brochu and colleagues $(8,12)$ focused on $\mathrm{BF}$ distribution, using WHR, and found significant changes in response to CET in male CAD subjects, but not in female, regardless of age, meaning there may be a gender effect. Our results contrast with these findings. Our study did not show any BF distribution changes in response to 12 months CET. The markers of BF distribution we used may not be sensible for detecting WHR changes, because the trunk ROI we analyzed includes, at least partly, the hip region and therefore may include the composition of both waist and hip circumferences measuring sites. On the other hand, Brochu and colleagues $(8,12)$ did not use other BF distribution markers, besides WHR, which, despite being a good surrogate for the risk of hazardous health outcomes (36) and particularly useful and easy usable in the clinical practice, have been shown to be both a poor surrogate marker for the distribution of BF and poorly correlated with DXA measurements $(23,24)$. These methodological differences limit the comparison or confrontation of our results with Brochu and colleagues work. The absence of changes in $\mathrm{BF}$ distribution found in our study mean that $\mathrm{BF}$ reduction must have occurred at an overall similar rate in all analyzed fat depots in response to our CET Program in CAD patients.

Many well-designed studies have reported BF distribution modifications in response to exercise (37-39). These studies, regardless of considerable dissimilarities between their interventions protocols, $\mathrm{BF}$ distribution markers and sample characteristics (gender, BMI, and age), have in common the fact that they all used interventions that included exercise and caused very significant negative energy balances. This is most definitely not yet a closed consensual topic, especially in CAD patients, and warrants further investigation. Despite the lack of studies on this topic in CAD patients, general measures of BF such as BMI and total BF has been shown to be better predictors of coronary risk factors than body fat distribution, in a sample of CAD patients (8) and so it is reasonable to expect that the significant reductions in these general BF markers may represent an overall health benefit and risk reduction for CAD patients $(2,16)$, even in the absence of changes in BF distribution, supporting the beneficial effect of the CET program intervention as compared with standard care.

Many aspects of body composition and, especially, of BF distribution remains to be studied and tested in CAD patients, particularly 
its response to specific aspects of exercise protocols. In order to test exercise safety, especially for higher exercise intensities, and increased effectiveness for achieving body composition and fat distribution changes in CAD patients it would be very interesting, in our point of view, to consider some recent findings when building new intervention' protocols for future trials. We suggest using higher exercise intensities in order to promote body composition and fat distribution changes, as tested in samples of subjects without cardiac conditions $(33,38)$

There are several strengths and limitations to this study. A major strength is the use of a long-term controlled intervention, as recommended by Kirk and colleagues (40) for exercise intervention studies with ad libitum diet to observe body composition responses. This study was performed on white male low-risk CAD patients only and the results should not be generalized. In this study caloric intake was not assessed and so, even with the long time span of the intervention and the presence of a control group results may still be influenced to some extent by subjects' diet. Nevertheless all subjects, from both groups, received the same dietary recommendations as part of their standard care follow-up. Subjects' group assignment was done based on residential proximity to exercise facilities, instead of random assignment which is gold standard to assure equal groups. Notwithstanding subjects were all from the same region (the metropolitan area of Lisbon city) differing only in few kilometer in the distance between subjects' residences and exercise facilities, additionally no differences were found between groups neither on studied nor on other variables in the baseline assessment and therefore we believe that no significant bias was introduced by the assignment strategy used. All subjects were also involved in phase II and III of cardiac rehabilitation which included some aerobic exercise for 6 months. This could have some impact on the results because some of the adaptations to exercise could have already taken place. However, it has been suggested that studies that use exercise without diet as the stimulus for weight loss should have at least 9-month duration to provide sufficient time for the full effects to occur (40). This oversize's the prior aerobic exercise period. Moreover it has been observed that CETprograms tend to have greater effect on the reduction of body fat than aerobic training alone, even in CAD patients $(17,18)$. Considering these reasons it is reasonable to hypothesize that the present CET program could result in changes in body composition and fat distribution. In this study, we used a limited amount of BF distribution variables based on DXA scans, and therefore our methodology excluded other commonly used BF distribution markers, such asWHR, and regional $\mathrm{BF}$ variables, such as visceral adipose tissue. Therefore, when we refer to our $\mathrm{BF}$ distribution results we are referring to the methodology used in this study.

The present study confirm the positive effect of a CET program on body composition of CAD patients, despite no changes in body mass or BMI. The absence of changes in BMI in subjects that changed their body composition recalls previously reported concerns and warns for the limitations of BMI for tracking body composition changes. It is not certain whether this CET program is suitable for resisting or delaying the natural effect of aging on regional $\mathrm{BF}$ and fat distribution. The fact that no alterations on $\mathrm{BF}$ distribution were observed means that fat reduction in all analyzed BF depots occurred at a similar rate in response to the intervention. $\mathrm{O}$

\footnotetext{
(c) 2012 The Obesity Society
}

\section{References}

1. Aronne LJ. Classification of obesity and assessment of obesity-related health risks. Obes Res 2002;10(Suppl 2):105S-115S.

2. Hubert HB, Feinleib M, McNamara PM, Castelli WP. Obesity as an independent risk factor for cardiovascular disease: a 26-year follow-up of participants in the Framingham Heart Study. Circulation 1983;67:968-977.

3. Kannel WB, Cupples LA, Ramaswami R et al. Regional obesity and risk of cardiovascular disease; the Framingham Study. J Clin Epidemiol 1991;44:183-190.

4. Larsson B, Svärdsudd K, Welin L et al. Abdominal adipose tissue distribution, obesity, and risk of cardiovascular disease and death: 13 year follow up of participants in the study of men born in 1913. Br Med J (Clin Res Ed) 1984;288:1401-1404.

5. Rossouw JE, Lewis B, Rifkind BM. The value of lowering cholesterol after myocardial infarction. $N$ Engl J Med 1990;323:1112-1119.

6. Albu JB, Lu J, Mooradian AD et al. Relationships of obesity and fat distribution with atherothrombotic risk factors: baseline results from the Bypass Angioplasty Revascularization Investigation 2 Diabetes (BARI 2D) trial. Obesity (Silver Spring) 2010;18:1046-1054

7. Hauner H, Stangl K, Schmatz C et al. Body fat distribution in men with angiographically confirmed coronary artery disease. Atherosclerosis 1990;85:203-210.

8. Brochu M, Poehlman ET, Savage P, Ross S, Ades PA. Coronary risk profiles in men with coronary artery disease: effects of body composition, fat distribution, age and fitness. Coron Artery Dis 2000;11:137-144.

9. AACVPR. Guidelines for Cardiac Rehabilitation and Secondary Prevention Programs. Human Kinetics: Champaign, 2004.

10. Balady GJ, Williams MA, Ades PA et al. Core components of cardiac rehabilitation/secondary prevention programs: 2007 update: a scientific statement from the American Heart Association Exercise, Cardiac Rehabilitation, and Prevention Committee, the Council on Clinical Cardiology; the Councils on Cardiovascular Nursing, Epidemiology and Prevention, and Nutrition, Physical Activity, and Metabolism; and the American Association of Cardiovascular and Pulmonary Rehabilitation. $J$ Cardiopulm Rehabil Prev 2007;27:121-129.

11. Donnelly JE, Blair SN, Jakicic JM et al. American College of Sports Medicine Position Stand. Appropriate physical activity intervention strategies for weight loss and prevention of weight regain for adults. Med Sci Sports Exerc 2009;41:459-471.

12. Brochu M, Poehlman ET, Savage P et al. Modest effects of exercise training alone on coronary risk factors and body composition in coronary patients. J Cardiopulm Rehabil 2000;20:180-188.

13. Brubaker PH, Rejeski WJ, Smith MJ et al. A home-based maintenance exercise program after center-based cardiac rehabilitation: effects on blood lipids, body composition, and functional capacity. J Cardiopulm Rehabil 2000;20:50-56.

14. Brubaker PH, Warner JG Jr, Rejeski WJ et al. Comparison of standard- and extended-length participation in cardiac rehabilitation on body composition, functional capacity, and blood lipids. Am J Cardiol 1996;78:769-773.

15. Lavie CJ, Milani RV. Effects of cardiac rehabilitation and exercise training in obese patients with coronary artery disease. Chest 1996;109:52-56.

16. Lavie CJ, Milani RV. Effects of cardiac rehabilitation, exercise training, and weight reduction on exercise capacity, coronary risk factors, behavioral characteristics, and quality of life in obese coronary patients. Am J Cardiol 1997;79:397-401.

17. Pierson LM, Herbert WG, Norton HJ et al. Effects of combined aerobic and resistance training versus aerobic training alone in cardiac rehabilitation. J Cardiopulm Rehabil 2001;21:101-110.

18. Santa-Clara H, Fernhall B, Baptista F, Mendes M, Bettencourt Sardinha L. Effect of a one-year combined exercise training program on body composition in men with coronary artery disease. Metab Clin Exp 2003;52:1413-1417.

19. Savage PD, Brochu M, Poehlman ET, Ades PA. Reduction in obesity and coronary risk factors after high caloric exercise training in overweight coronary patients. Am Heart J 2003;146:317-323.

20. Park YW, Heymsfield SB, Gallagher D. Are dual-energy X-ray absorptiometry regional estimates associated with visceral adipose tissue mass? Int $J$ Obes Relat Metab Disord 2002;26:978-983.

21. Pimenta N, Santa-Clara H, Fragoso IJ. Comparison of body composition and body fat distribution of patients following a cardiac rehabilitation program and sedentary patients. Rev Port Cardiol 2010;29:1163-1180.

22. Lohman TG, Roche AF, Martorell R. Anthropometric Standardization Reference Manual. Human Kinetics: Champaign, 1988.

23. Savgan-Gurol E, Bredella M, Russell M et al. Waist to hip ratio and trunk to extremity fat (DXA) are better surrogates for IMCL and for visceral fat respectively than for subcutaneous fat in adolescent girls. Nutr Metab (Lond) 2010;7:86.

24. Ketel IJ, Volman MN, Seidell JC et al. Superiority of skinfold measurements and waist over waist-to-hip ratio for determination of body fat distribution in a population-based cohort of Caucasian Dutch adults. Eur J Endocrinol 2007;156:655-661.

25 . Bruce RA. Exercise testing of patients with coronary heart disease. Principles and normal standards for evaluation. Ann Clin Res 1971;3:323-332.

26. Karvonen MJ, Kentala E, Mustala O. The effects of training on heart rate; a longitudinal study. Ann Med Exp Biol Fenn 1957;35:307-315.

27. Brochu M, Poehlman ET, Ades PA. Obesity, body fat distribution, and coronary artery disease. J Cardiopulm Rehabil 2000;20:96-108.

28. Garn SM, Leonard WR, Hawthorne VM. Three limitations of the body mass index. Am J Clin Nutr 1986;44:996-997. 
29. Hortobágyi T, Israel RG, O'Brien KF. Sensitivity and specificity of the Quetelet index to assess obesity in men and women. Eur J Clin Nutr 1994;48:369-375.

30. Volaklis KA, Spassis AT, Tokmakidis SP. Land versus water exercise in patients with coronary artery disease: effects on body composition, blood lipids, and physical fitness. Am Heart $J$ 2007; 154:560.e1-560.e6.

31. Goodpaster BH, Delany JP, Otto AD et al. Effects of diet and physical activity interventions on weight loss and cardiometabolic risk factors in severely obese adults: a randomized trial. JAMA 2010;304:1795-1802.

32. Sillanpää E, Häkkinen A, Nyman K et al. Body composition and fitness during strength and/or endurance training in older men. Med Sci Sports Exerc 2008;40: 950-958.

33. Irving BA, Davis CK, Brock DW et al. Effect of exercise training intensity on abdominal visceral fat and body composition. Med Sci Sports Exerc 2008;40: 1863-1872.

34. Enzi G, Gasparo M, Biondetti PR et al. Subcutaneous and visceral fat distribution according to sex, age, and overweight, evaluated by computed tomography. Am J Clin Nutr 1986;44:739-746.
35. Fantin F, Di Francesco V, Fontana G et al. Longitudinal body composition changes in old men and women: interrelationships with worsening disability. J Gerontol A Biol Sci Med Sci 2007;62:1375-1381.

36. Nishida C, Ko GT, Kumanyika S. Body fat distribution and noncommunicable diseases in populations: overview of the 2008 WHO Expert Consultation on Waist Circumference and Waist-Hip Ratio. Eur J Clin Nutr 2010;64:2-5.

37. Mayo MJ, Grantham JR, Balasekaran G. Exercise-induced weight loss preferentially reduces abdominal fat. Med Sci Sports Exerc 2003;35:207-213.

38. Arciero PJ, Gentile CL, Martin-Pressman R et al. Increased dietary protein and combined high intensity aerobic and resistance exercise improves body fat distribution and cardiovascular risk factors. Int J Sport Nutr Exerc Metab 2006;16:373-392.

39. Redman LM, Heilbronn LK, Martin CK et al. Effect of calorie restriction with or without exercise on body composition and fat distribution. J Clin Endocrinol Metab 2007;92:865-872.

40. Kirk EP, Jacobsen DJ, Gibson C, Hill JO, Donnelly JE. Time course for changes in aerobic capacity and body composition in overweight men and women in response to long-term exercise: the Midwest Exercise Trial (MET). Int J Obes Relat Metab Disord 2003;27:912-919. 